1. Derjaguin, B. V. \& Landau, L. Theory of the stability of strongly charged lyophobic sols and the adhesion of strongly charged particles in solutions of electrolytes. Acta Physicochimica (USSR) 14, 633 (1941).

2. Verwey, E. J. \& Overbeek, J. Th. G. Theory of the Stability of Lyophobic Colloids (Elsevier, Amsterdam, 1948).

3. Crocker, J. C. \& Grier, D. G. Microscopic measurements of pair interaction potential of chargestabilised colloid. Phys. Rev. Lett. 73, 352-355 (1994)

4. Crocker, J. C. \& Grier, D. G. Methods of digital video microscopy for colloidal studies. J. Coll. Interface Sci. 179, 298-310 (1996).

5. Grier, D. G. Optical tweezers in colloid and interface science. Curr. Opin. Coll. Interface Sci. 2, 264-270 (1997).

6. Larsen, A. E. \& Grier, D. G. Like-charge attractions in metastable colloidal crystallites. Nature $\mathbf{3 8 5}$ 230-233 (1997)

7. Ise, N., Okubo, T., Sugimura, M., Ito, K. \& Nolte, H. J. Ordered structure in dilute solutions of highly charge polymers lattices as studied by microscopy. I. Interparticle distance as a function of latex concentration. J. Chem. Phys. 78, 536-540 (1983).

8. Kepler, G. M. \& Fraden, S. Attractive potential between confined colloids at low ionic strength. Phys. Rev. Lett. 73, 356-359 (1994).

9. Carbajal-Tinoco, M. D., Castro-Roman, F. \& Arauz-Lara, J. L. Static properties of confined colloidal suspensions. Phys. Rev. E 53, 3745-3749 (1996).

10. Crocker, J. C. \& Grier, D. G. When like charge particles attract: the effects of geometrical confinement on long-range colloidal interactions. Phys. Rev. Lett. 77, 1897-1900 (1996).

11. Sogami, I. Effect potential between charged spherical particle in dilute suspension. Phys. Lett. A 96, 199-203 (1983).

12. Sogami, I. \& Ise, N. On the electrostatic interaction in macroionic solutions. J. Chem. Phys. 81, 63206332 (1984).

13. Chu, X. \& Wasan, D. Attractive interaction between similarly charged colloidal particles. J. Coll. Interface. Sci. 184, 268-278 (1996).

14. Jönsson, B., Åkesson, T. \& Woodward, C. E. in Ordering and Phase Transitions in Colloidal Systems (eds Arora, A. K. \& Tata, B. V. R.) Ch. 11, 295-313 (VCH, New York, 1996).

15. Smalley, M. V. in Ordering and Phase Transitions in Colloidal Systems (eds Arora, A. K. \& Tata, B. V. R.) Ch. 12, 315-337 (VCH, New York, 1996)

16. Bowen, W. R. \& Sharif, A. O. Adaptive finite element solution of the non-linear Poisson-Boltzmann equation: a charged spherical particle at various distances from a charged cylindrical pore in a charged planar surface. J. Coll. Interface Sci. 187, 363-374 (1997).

17. Russel, W. B., Saville, D. A. \& Schowalter, W. R. Colloidal Dispersions (Cambridge Univ. Press, 1989) 18. Hastings, R. On the crystallisation of macroionic solutions. J. Chem. Phys. 68, 675-678 (1978).

19. van Roij, R. \& Hansen, J-P. Van der Waals-like instability is suspensions of mutually repelling charged colloids. Phys. Rev. Lett. 79, 3082-3085 (1997)

20. Tata, B. V. R., Yamahara, E., Rajamani, P. V. \& Ise, N. Amorphous clustering in highly charged dilute poly(chlorostyrene-styrene sulfonate) colloids. Phys. Rev. Lett. 78, 2660-2663 (1997).

Acknowledgements. This work was funded by the UK Biotechnology and Biological Sciences Research Council.

Correspondence should be addressed to W.R.B. (e-mail: r.bowen@swansea.ac.uk).

\section{Non-volatile holographic storage in doubly doped lithium niobate crystals}

\author{
K. Buse, A. Adibi \& D. Psaltis \\ California Institute of Technology, Department of Electrical Engineering, \\ Pasadena, California 91125, USA
}

Photorefractive materials are being widely investigated for applications in holographic data storage ${ }^{1}$. Inhomogeneous illumination of these materials with an optical interference pattern redistributes charge, builds up internal electric fields and so changes the refractive index. Subsequent homogeneous illumination results in light diffraction and reconstructs the information encoded in the original interference pattern. A range of inorganic and organic photorefractive materials are $k n w^{2}$, in which thousands of holograms of high fidelity can be efficiently stored, reconstructed and erased. But there remains a problem with volatility: the read-out process usually erases the stored information and amplifies the scattered light. Several techniques for 'fixing' holograms have been developed ${ }^{3-6}$, but they have practical disadvantages and only laboratory demonstrators have been built $^{7-10}$. Here we describe a resolution to the problem of volatility that should lead to the realization of a more practical system. We use crystals of lithium niobate-available both in large size and with excellent homogeneity - that have been doped with two different deep electron traps (iron and manganese). Illumination of the crystals with incoherent ultraviolet light during the record- a

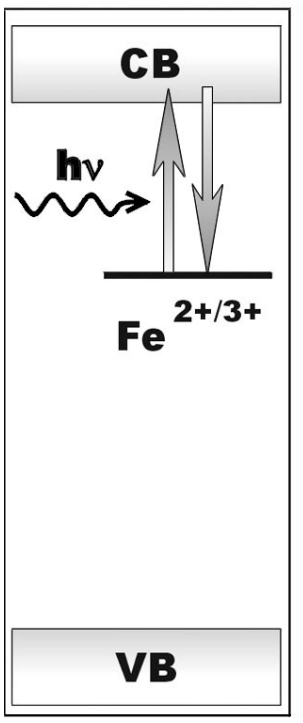

b

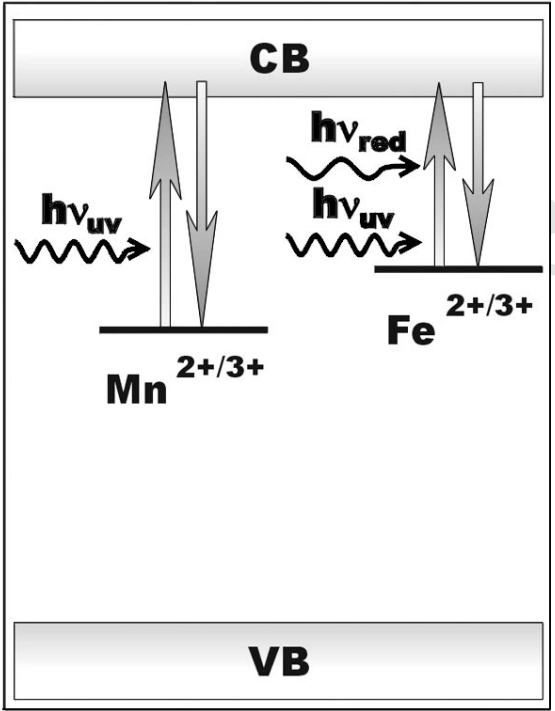

Figure 1 Band diagram of $\mathrm{LiNbO}_{3}$ doped with iron (a) and doubly doped with iron and manganese (b). CB, conduction band; VB, valence band. Manganese has a deeper energy level than iron. Both elements occur in two different valence states. Electrons are excited into the conduction band from $\mathrm{Fe}^{2+}$ either by visible light or by ultraviolet light, or from $\mathrm{Mn}^{2+}$ by ultraviolet light only. Conduction-band electrons can recombine with $\mathrm{Fe}^{3+}$ or with $\mathrm{Mn}^{3+}$

ing process permits the storage of data (a red-light interference pattern) that can be subsequently read, in the absence of ultraviolet light, without erasure. Our crystals show up to 32 per cent diffraction efficiency, rapid optical erasure of the stored data is possible using ultraviolet light, and light scattering is effectively prevented.

Build-up of space-charge fields in photorefractive materials requires redistribution of charge. Transition-metal ions can occur in inorganic crystals in different valence states: for example, iron is present in $\mathrm{LiNbO}_{3}$ as $\mathrm{Fe}^{2+}$ and $\mathrm{Fe}^{3+}$ (ref. 11). The energy level of such a centre is located in the gap between valence and conduction band; Fig. 1a illustrates this. Iron is a deep centre, and hence thermal excitations are negligible. Light, however, excites electrons from $\mathrm{Fe}^{2+}$, the electrons migrate in the conduction band and finally they are trapped somewhere else by $\mathrm{Fe}^{3+}$ sites. The dominant charge driving force in $\mathrm{LiNbO}_{3}$ is the bulk photovoltaic effect, which causes the excited electrons to migrate in a preferred direction. Drift and diffusion can also contribute to the movement of the charge carriers. Reconstruction of stored information, which requires homogeneous illumination with light of the recording wavelength, causes uniform electron excitation. As a result, the charge pattern is 'washed out' and the hologram becomes erased.

One approach to obtaining non-volatile storage is to copy the space-charge pattern into a pattern of immobile ions ${ }^{3}$ ('thermal fixing'). Ions become mobile if the crystals are heated; they migrate to compensate the space-charge field and after cooling they become immobile again. The resulting ion pattern cannot be erased by light. Alternatively, the recorded space-charge field can be copied into ferroelectric domains ${ }^{4}$ ('electrical fixing'). This is accomplished through application of external electric fields, which switch ferroelectric domains in the regions where the external and internal fields add up. The domain pattern is also stable against further illumination. Unfortunately, heating or application of large external fields is not practical and rapid refreshing of the memory is not possible. Two-step recording ${ }^{5}$ is an all-optical approach for non-volatile storage: recording light of low photon energy and sensitizing light 
of high photon energy excite electrons via virtual or real intermediate levels to the conduction band. For read-out only, light of low photon energy is used which cannot by itself generate free electrons, and thus the stored charge pattern is stable. Despite impressive recent progress ${ }^{12}$, the low sensitivity of this approach remains a serious drawback. Recording with light of one wavelength and just reading with light of a longer one causes substantial information losses due to the Bragg condition of volume diffraction ${ }^{6}$. Even though all these techniques work ${ }^{7-10}$, the outlined disadvantages have made holographic data storage impractical thus far.

The method we describe here uses crystals that have two different deep traps, with energy levels in the gap between the valence and conduction bands. Such crystals are often photochromic ${ }^{13,14}$, which means that the absorption of the material at one wavelength can be changed by illuminating it at another wavelength. We can explain the physical mechanism for photochromism in such doubly doped crystals with reference to Fig. $1 \mathrm{~b}$, which is the band diagram for a $\mathrm{LiNbO}_{3}$ crystal doped with iron (shallower trap) and manganese (deeper trap). Manganese occurs in $\mathrm{LiNbO}_{3}$ in the valence states $2+$ and $3+$ (ref. 15). Initially, the electrons tend to be in the deeper traps and the crystal is transparent for wavelengths corresponding to the $\mathrm{Fe}^{2+/ 3+}$ level (centred at $477 \mathrm{~nm}$ ). If the crystal is illuminated with light at shorter wavelengths that can ionize the deeper manganese traps, then the iron traps become populated and the crystal becomes absorptive for a wide range of visible wavelengths. We can return the crystal to its transparent state by illuminating it with light in the visible range, which transfers the electrons from the iron traps back to the manganese traps. This is shown in Fig. 2, which is a photograph of such a crystal after ultraviolet pre-exposure and subsequent red-light illumination. The red light was present in the centre of the crystal only, and the bleaching can be clearly seen. It has been previously observed that the holographic sensitivity of such photochromic photorefractive crystals is enhanced for longer wavelengths when they are pre-exposed to short-wavelength radiation $^{13,16}$. Here we show how we can use photochromic

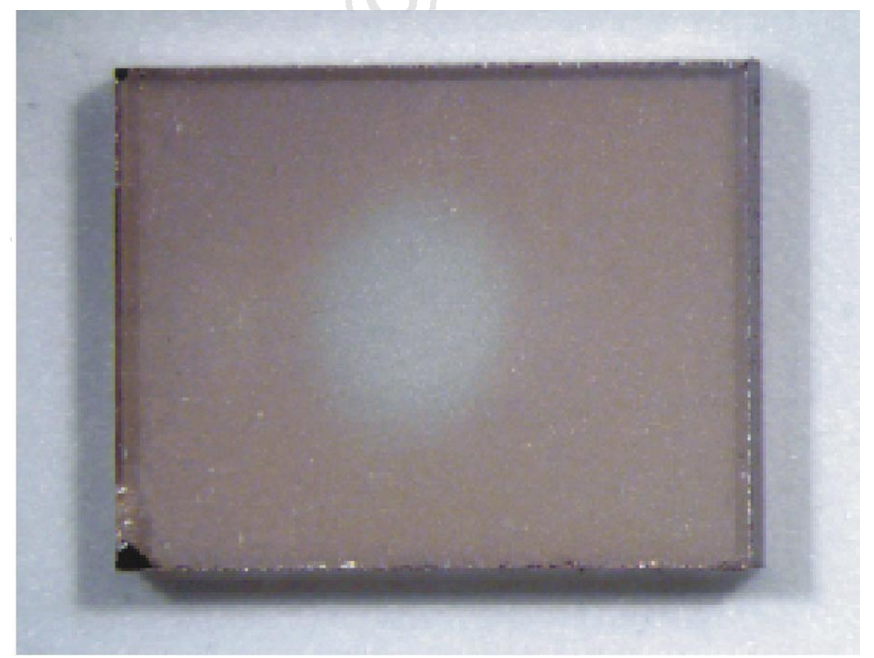

Figure 2 Photo-assisted electron transfer between traps in doubly doped $\mathrm{LiNbO}_{3}$. Photograph of a $\mathrm{LiNbO}_{3}$ crystal (dimensions $5 \times 4 \times 0.85 \mathrm{~mm}^{3}$ ) doped with $0.075 \mathrm{wt} \% \mathrm{Fe}_{2} \mathrm{O}_{3}$ and $0.01 \mathrm{wt} \% \mathrm{MnO}$ after homogeneous illumination with ultraviolet light (wavelength $365 \mathrm{~nm}$, intensity $20 \mathrm{~mW} \mathrm{~cm}^{-2}, 120 \mathrm{~min}$ ) and subsequent illumination of the middle of the crystal face with a laser light beam (wavelength $633 \mathrm{~nm}$, intensity $2,400 \mathrm{~mW} \mathrm{~cm}^{-2}, 1 / \mathrm{e}^{2}$ diameter $1 \mathrm{~mm}, 30 \mathrm{~min}$ ). The ultraviolet light excites electrons from manganese into iron, creating $\mathrm{Fe}^{2+}$. This centre absorbs visible light and the crystal is darkened. The red light excites the electrons from iron and they are trapped in manganese, where they cannot be excited by visible light. Thus the crystal is bleached by the red light. doubly doped $\mathrm{LiNbO}_{3}$ crystals to realize non-volatile holographic storage.

Experiments were performed with a $0.85-\mathrm{mm}$-thick $\mathrm{LiNbO}_{3}$ crystal doped with 0.075 wt $\% \mathrm{Fe}_{2} \mathrm{O}_{3}$ and $0.01 \mathrm{wt} \% \mathrm{MnO}$. We used a 100-W mercury lamp as the ultraviolet light source, and a $35-\mathrm{mW}$ $\mathrm{HeNe}$ laser for holographic recording. The unpolarized ultraviolet light illuminates the crystal evenly (wavelength $365 \mathrm{~nm}$, intensity $20 \mathrm{~mW} \mathrm{~cm}^{-2}$ ); the HeNe laser light is split into two plane waves which interfere at the crystal (wavelength $633 \mathrm{~nm}$, beam diameter $2.0 \mathrm{~mm}$, intensity of each wave $300 \mathrm{~mW} \mathrm{~cm}^{-2}$, transmission geometry; quoted diameter and intensity are for the area within which power drops to $1 / \mathrm{e}^{2}$ of its maximum value). The grating vector of the interference pattern is aligned along the $c$ axis of the sample. During recording, one of the HeNe beams is blocked from time to time and the second beam is diffracted from the written grating to obtain the diffraction efficiency $\eta$ as the ratio between diffracted and total incident light powers.

The lower curve in Fig. 3 shows the evolution of the diffraction efficiency when a holographic grating is recorded with the $\mathrm{HeNe}$ laser light beams only, following a 2-hour pre-exposure to ultraviolet light. It increases rapidly, reaches a maximum and decreases afterwards almost to zero. The origin of this behaviour is clear: the ultraviolet pre-exposure excites electrons from manganese centres and populates homogeneously the iron level. As the $\mathrm{Fe}^{2+}$ ions can absorb red light, the HeNe laser records a hologram. Interference maxima yield large photovoltaic currents, which build up spacecharge fields and refractive-index changes. However, the $\mathrm{Fe}^{2+}$ sites become bleached in the high-intensity regions and the current drops. Ultimately, the darker regions are also bleached, and all electrons are trapped by the $\mathrm{Mn}^{3+}$ ions. However, the achieved $\mathrm{Mn}^{2+}$ concentration is almost completely spatially homogeneous; this is because we start with a homogeneous $\mathrm{Fe}^{2+}$ concentration, and each excited carrier is moved in the same direction by approximately the same distance before it becomes retrapped by the $\mathrm{Mn}^{3+}$ ions. Thus the final space-charge field is very small, and this approach is not suitable for efficient storage.

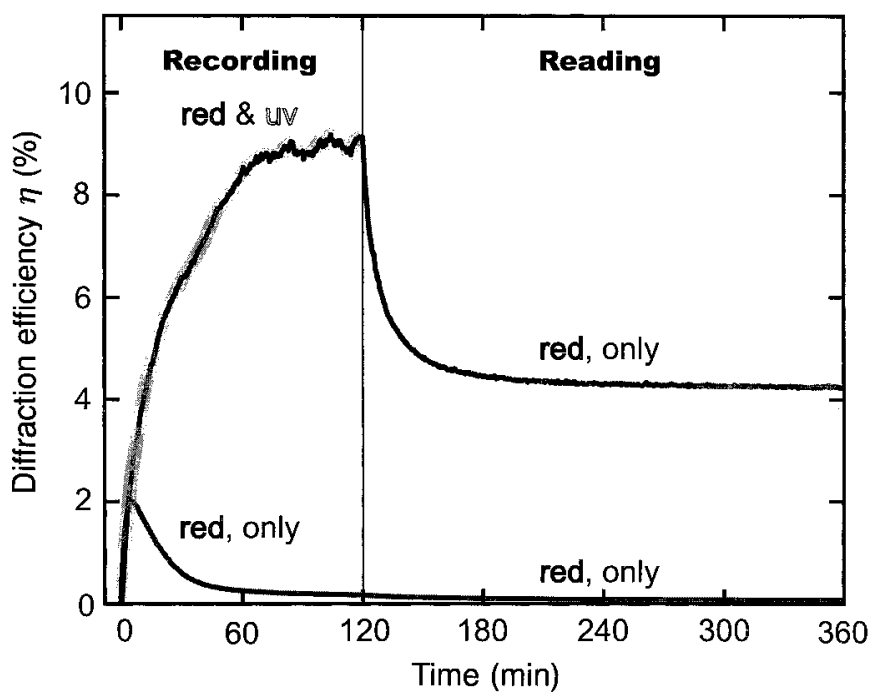

Figure 3 Holographic recording and read-out curves. The diffraction efficiency $\eta$, the ratio between the intensity of the diffracted and of the total incident read-out light, is shown against time. Lower curve: the crystal is pre-exposed to the ultraviolet light (wavelength $365 \mathrm{~nm}$, intensity $20 \mathrm{~mW} \mathrm{~cm}^{-2}, 120 \mathrm{~min}$ ), then the hologram is recorded (wavelength $633 \mathrm{~nm}$, ordinary polarization, intensity $600 \mathrm{~mW} \mathrm{~cm}^{-2}, 120 \mathrm{~min}$ ) and finally it is read by one of the recording beams (633 nm, intensity $300 \mathrm{~mW} \mathrm{~cm}^{-2}, 240 \mathrm{~min}$ ). Upper curve: same procedure, but the ultraviolet light is also present during recording. Simultaneous presence of ultraviolet and red light yields higher efficiencies and in the final stage, nonvolatile read-out. 
The central idea that allows non-volatile storage is to illuminate the crystal with ultraviolet and red light simultaneously, wait until saturation is reached and then switch the ultraviolet light off. The upper curve in Fig. 3 shows the result obtained by this method. The first observation is that much larger efficiencies are achieved during recording. In addition, read-out erases only a fraction of the hologram because electrons are removed from iron. However, after complete bleaching of the $\mathrm{Fe}^{2+}$ sites, the hologram remains recorded in the manganese traps. From this stage on, the crystal is insensitive to red light and read-out is non-volatile. Extrapolation of a 12-hour reading experiment shows that continuous read-out of at least two weeks is possible until the diffraction efficiency drops further by a factor of $1 / \mathrm{e}$.

Figure 4 illustrates the processes schematically. The light intensities and the concentrations of electrons trapped in iron and manganese are shown against the spatial coordinate before, during and after recording. At the beginning, only homogeneous ultraviolet light is present (Fig. 4a). Some electrons are trapped in manganese and some in iron.

During recording (Fig. $4 \mathrm{~b}$ ), the presence of the red interference pattern causes electrons to be excited from iron and moved back to manganese in the regions of interference maxima. The bulk photovoltaic currents, which are proportional to the product of light
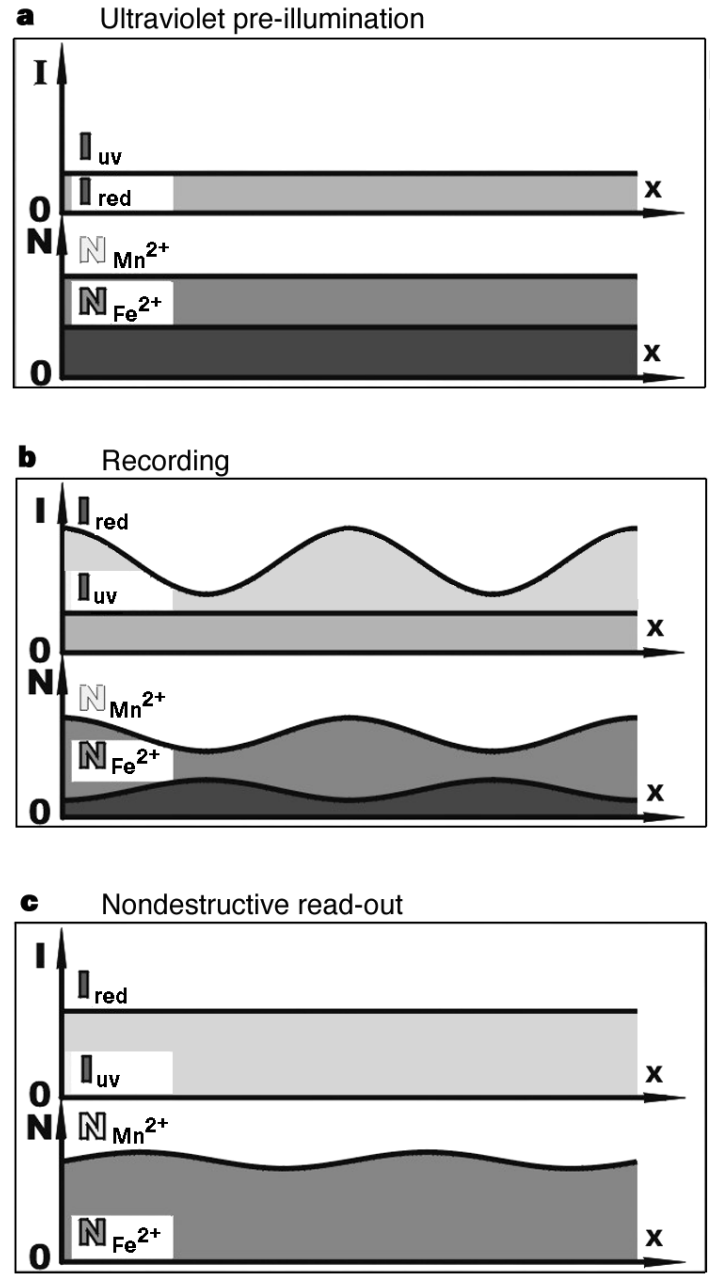

Figure 4 Recording and non-destructive read-out processes. Intensities of red and ultraviolet light ( $/$ red and $/$ uv $)$, and concentrations of electrons trapped in iron and manganese $\left(N_{\mathrm{Fe}^{2+}}\right.$ and $\left.N_{\mathrm{Mn}^{2+}}\right)$ against spatial coordinate $x$ along the grating vector during ultraviolet pre-illumination (a), recording (b) and non-destructive read-out $(\mathbf{c})$. intensity and concentration of filled electron traps, create spatially inhomogeneous currents that contribute to build-up of the spacecharge field. Homogeneous ultraviolet light excites electrons from the modulated iron and manganese patterns, while modulated red light excites electrons only from iron. As the excited electron distribution and current pattern are replicas of the red-light intensity pattern, the distribution of the charge re-trapped in manganese also stores the same pattern. The continuous presence of ultraviolet light replenishes the iron sites and makes it possible to sustain and build up the transfer of information from iron to manganese. Detailed analysis of this process yields the following result: saturation space-charge field, refractive-index change and diffraction efficiency depend on the ratio of the intensities of the red and ultraviolet light, and not on their absolute values. Red light of too high an intensity bleaches the $\mathrm{Fe}^{2+}$ sites, the concentration of electrons in iron is small, and the space-charge field approaches zero. If the red light is too weak, the ultraviolet light erases the information, and the space-charge field again becomes zero.

After recording, the ultraviolet light is switched off. The red light initially removes the electrons from iron until all of them are trapped in manganese, and read-out becomes non-volatile (Fig. 4c). However, optical erasure by homogeneous ultraviolet light is still possible.

Our experiments reach a non-volatile diffraction efficiency of $4 \%$ for ordinarily polarized light and, due to a larger electro-optic coefficient, $32 \%$ for extraordinary polarization. The square root of the saturation efficiency yields the quantity $M / \#$, a measure of how many holograms can be multiplexed ${ }^{17}$. We get a maximum $M / \#$ of $\sim 0.6$ with the $0.85-\mathrm{mm}$-thick crystal. Typical values for volatile recording with green light in iron-doped material are around $M / \#=1$ for $\sim 1$-cm-thick crystals ${ }^{17}$. As $M / \#$ is proportional to the thickness of the recording material, a $1-\mathrm{cm}$-thick crystal with the new technique should yield $M / \# \approx 7$. The areal density of absorbed light energy required to get $1 \%$ diffraction efficiency is denoted by $W_{\eta=1 \%}$. Our non-volatile method requires $W_{\eta=1 \%}=3.5 \mathrm{~J} \mathrm{~cm}^{-2}$ of red light, while typical values achieved with green light in irondoped material are $\sim 1 \mathrm{~J} \mathrm{~cm}^{-2}$. For instance, for a recording area of $1 \mathrm{~mm}^{2}$, and $10 \mathrm{~mW}$ of absorbed red light, the exposure time required to achieve diffraction efficiency equal to $10^{-6}$ is less than $35 \mathrm{~ms}$, corresponding to a recording rate of $\sim 30 \mathrm{Mbits}^{-1}$. It is remarkable that the new technique provides a significant improvement in $M / \#$ and does not sacrifice sensitivity $\left(W_{\eta=1 \%}\right)$, even though it was produced in order to provide convenient non-volatile storage and its parameters here not yet been optimized. For instance, the concentrations of iron and manganese can be fine-tuned. Use of cerium instead of iron, and copper instead of manganese, may further improve both $M / \#$ and $W_{\eta=1 \%}$. A change of the wavelength of the sensitizing light may enable additional advances to be made.

The main advantages of our technique compared to other nonvolatile storage methods are that there is no need for external electric fields or light of high intensity. Furthermore, during recording, the ultraviolet light prevents build-up of both holographically amplified scattered light and screening fields created by accumulation of charge at the boundaries of illuminated regions. During read-out, the crystal is insensitive and cross-talk build-up due to two-wave mixing effects does not occur. Thus the fidelity of the stored information is significantly improved and the error rate drops. Smooth and continuous recording curves like those shown in Fig. 3 are hard to obtain in conventional experiments. It appears that we have discovered a recording method in which essentially all the desirable features of holographic memory are improved. We consider that the prospects are excellent for practical holographic storage devices using this technique.

Received 4 February; accepted 22 April 1998.

1. Psaltis, D. \& Mok, F. Holographic memories. Sci. Am. 273, 70-76 (1995).

2. Meerholz, K., Volodin, B. L., Sandalphon, Kippelen, B. \& Peyghambarian, N. A photorefractive polymer with high optical gain and diffraction efficiency near 100\%. Nature 371, 497-500 (1994). 
3. Amodei, J. J. \& Staebler, D. L. Holographic pattern fixing in electro-optic crystals. Appl. Phys. Lett. 18, 540-542 (1971)

4. Micheron, F. \& Bismuth, G. Electrical control of fixation and erasure of holographic patterns in ferroelectric materials. Appl. Phys. Lett. 20, 79-81 (1972).

5. von der Linde, D., Glass, A. M. \& Rodgers, K. F. Multiphoton photorefractive processes for optical storage in $\mathrm{LiNbO}_{3}$. Appl. Phys. Lett. 25, 155-157 (1974).

6. Külich, H. C. A new approach to read volume holograms at different wavelengths. Opt. Commun. 64, 407-411 (1987).

7. Heanue, J. F., Bashaw, M. C., Daiber, A. J., Snyder, R. \& Hesselink, L. Digital holographic storage system incorporating thermal fixing in lithium niobate. Opt. Lett. 21, 1615-1617 (1996).

8. Ma, J. et al. Electrical fixing of 1000 angle-multiplexed holograms in SBN:75. Opt. Lett. 22, 1116-1118 (1997).

9. Lande, D., Orlov, S. S., Akella, A., Hesselink, L. \& Neurgaonkar, R. R. Digital holographic storage system incorporating optical fixing. Opt. Lett. 22, 1722-1724 (1997).

10. Chuang, E. \& Psaltis, D. Storage of 1000 holograms with use of a dual-wavelength method. Appl. Opt. 36, 8445-8454 (1997)

11. Kurz, H. et al. Photorefractive centers in $\mathrm{LiNbO}_{3}$ studied by optical, Mössbauer- and EPR-methods. Appl. Phys. 12, 355-368 (1977).

12. Guenther, H., Wittmann, G., Macfarlane, R. M. \& Neurgaonkar, R. R. Intensity dependence and white-light gating of two-color photorefractive gratings in $\mathrm{LiNbO}_{3}$. Opt. Lett. 22, 1305-1307 (1997)

13. Staebler, D. L. \& Phillips, W. Hologram storage in photochromic $\mathrm{LiNbO}_{3}$. Appl. Phys. Lett. 24, 268 270 (1974)

14. Ming, Y., Krätzig, E. \& Orlowski, R. Photorefractive effects in $\mathrm{LiNbO}_{3}: \mathrm{Cr}$ induced by two-step excitations. Phys. Status Solidi A 92, 221-229 (1985).

15. Thiemann, O. \& Schirmer, O. F. Energy levels of several $3 \mathrm{~d}$ impurities and $\mathrm{EPR}$ of $\mathrm{Ti}^{3+}$ in $\mathrm{LiNbO}_{3}$. Proc. SPIE 1018, 18-22 (1988).

16. Buse, K., Holtmann, L. \& Krätzig, E. Activation of $\mathrm{BaTiO}_{3}$ for infrared holographic recording. Opt Commun. 85, 183-186 (1991).

17. Mok, F. H., Burr, G. W. \& Psaltis, D. System metric for holographic memory systems. Opt. Lett. 21, 896-898 (1996).

Acknowledgements. We thank E. Krätzig for discussions. This work was supported by JPL, funded by DARPA/ITO, and by grants from Rome Labs. K.B. thanks the Deutsche Forschungsgemeinschaft for a postdoctoral fellowship.

Correspondence and requests for materials should be addressed to D.P. (e-mail: psaltis@sunoptics. caltech.edu)

\section{Isolation and properties of small-bandgap fullerenes}

\section{Michael D. Diener \& John M. Alford}

TDA Research Inc., 12345 West 52nd Avenue, Wheat Ridge, Colorado 80033, USA

The diversity of molecular structures exhibited by fullerenes ${ }^{1}$ suggests a wide range of interesting and useful properties. Several fullerenes are now considered to be well characterized, but only minor variations in their chemical and physical properties have been observed ${ }^{2}$. Here we show that there are in fact two distinct classes of fullerenes, with some very different chemical properties. Members of the first class, typified by $\mathrm{C}_{60}$ and $\mathrm{C}_{70}$, have large energy gaps between the highest occupied and lowest unoccupied molecular orbitals (HOMO and LUMO), and are soluble in many organic solvents. The second, previously unrecognized class is represented by $\mathrm{C}_{74}$ and selected isomers of the higher fullerenes, such as that of $\mathrm{C}_{80}$ with icosahedral symmetry: these are either free radicals or have small HOMO-LUMO gaps. Like radical metallofullerenes, they are kinetically unstable and react readily to form insoluble, polymerized solids. These intermolecular bonds can be broken by electrochemical reduction. After reducing them to soluble anions, we have been able to isolate and characterize these new fullerenes.

The solubility of fullerenes in common organic solvents is a primary factor contributing to the rapid advancement of fullerene chemistry. Solvation allows extraction of fullerene clusters from carbon $\operatorname{soot}^{3}$, isolation of individual structures, and derivatization to form new compounds. Isomers of $\mathrm{C}_{60}$ through to $\mathrm{C}_{96}$ have been isolated and identified ${ }^{4-6}$, and these extractable isomers share common electronic features ${ }^{7}$. Specifically, both experimental ${ }^{8-10}$ and computational ${ }^{11,12}$ investigations show that all soluble fullerenes have large bandgaps. As small-bandgap and radical fullerene structures were not identified in the extracts, it was generally concluded that these isomers were thermally annealed into more stable structures and were not present in the soot.
$\mathrm{C}_{74}$ is an anomaly because it is regularly observed in soots by mass spectrometry, but it is not soluble. Like $\mathrm{C}_{60}$ and $\mathrm{C}_{70}, \mathrm{C}_{74}$ possesses only one possible isolated pentagon structure. Previous low-level Hückel molecular orbital ${ }^{1,11}$ and tight-binding molecular dynamics calculations ${ }^{7,12}$ on $\mathrm{C}_{74}\left(D_{3}\right)$ predicted a small bandgap, and it has been suggested that this could explain its insolubility ${ }^{7}$. More accurate density functional theory calculations (Fig. 1) confirm a very small $(0.05 \mathrm{eV})$ bandgap, and thermal population of the triplet state will produce a chemically reactive biradical. Adding two electrons to $\mathrm{C}_{74}$ creates a kinetically stable (and soluble) closedshell configuration with a moderate bandgap. An example is Ca@ $\mathrm{C}_{74}$ (ref. 13), a soluble endohedral metallofullerene in which two electrons have been donated from the $\mathrm{Ca}$ atom, filling the LUMO.

Observations are similar for the higher fullerenes. Only a small percentage of the $\mathrm{C}_{80}$ found in soots can be solvent-extracted, and this large-bandgap $D_{2}$ isomer ${ }^{14}$ may not be as thermodynamically stable as the $D_{5 h}$ or $I_{h}$ radical isomers ${ }^{12,15}$. As the undistorted ground state HOMO of $\mathrm{C}_{80}\left(I_{h}\right)$ has only two electrons in four orbitals, the addition of six electrons produces a closed-shell, large-bandgap species, explaining the solubility of $\mathrm{La}_{2} @ \mathrm{C}_{80}$ (ref. 16).

It is likely that larger fullerenes $\left(>C_{100}\right)$ consist almost entirely of insoluble small-bandgap structures. Calculations indicate that the statistical probability of forming closed-shell, large-bandgap structures past $\mathrm{C}_{100}$ is very small ${ }^{1}$, and this could account for the large amount of insoluble giant fullerenes observed in the soots. A report $^{17}$ demonstrating that giant fullerenes (as well as $\mathrm{C}_{74}$ and $\mathrm{C}_{80}$ ) can be extracted after chemical derivatization to passivate radical structures strongly suggests that they too have radical or small-bandgap configurations. Although we have considered only isolated pentagon isomers with small bandgaps, the soot could also contain adjacent pentagon isomers which are highly reactive owing to excessive pyramidalization (steric strain) along the pentagon junctions ${ }^{31}$.

With small-bandgap (and radical) electronic structures, $\mathrm{C}_{74}$ and $\mathrm{C}_{80}\left(I_{h}\right)$ exhibit properties similar to the endohedral lanthanide metallofullerenes. The majority of radical Ln-metallofullerenes are

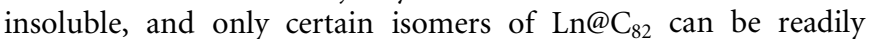
extracted $^{18}$. The unique solubility of $\mathrm{Ln}_{0} \mathrm{C}_{82}$ remains a mystery, but it is probably due to localization of the radical electron in bonds near the Ln atom. In addition to their similar electronic configurations, the unfilled orbitals of both small-bandgap fullerenes

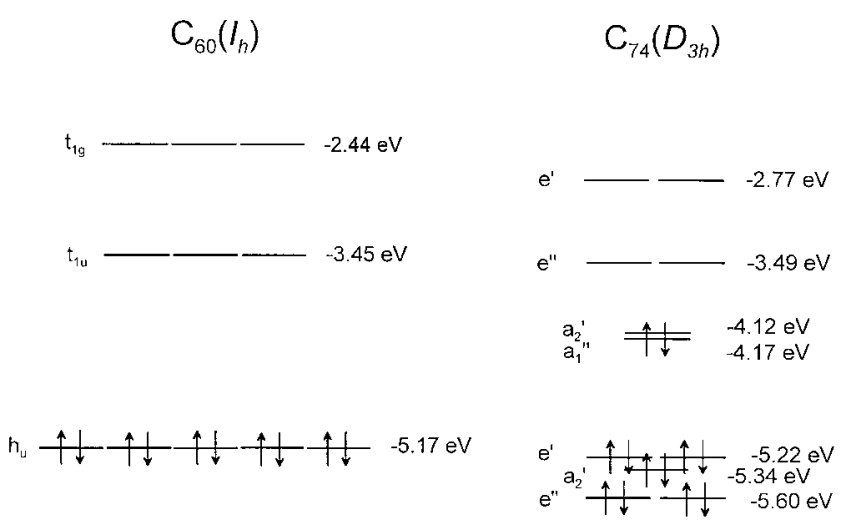

Figure 1 Kohn-Sham orbital energy levels of $\mathrm{C}_{60}$ and $\mathrm{C}_{74}$. Density functional theory calculations were performed using the BLYP functional in Gaussian 94 (ref. 28). Geometries were optimized using the $3-21 G^{*}$ basis set, followed by single point calculations using the $6-31 G^{*}$ basis set. The calculated value of $1.72 \mathrm{eV}$ for the $\mathrm{C}_{60}$ bandgap is in good agreement with a previous calculation of $1.64 \mathrm{eV}$ (ref. 29). A singlet state was assumed for $C_{74}$. 